This item was submitted to Loughborough's Research Repository by the author.

Items in Figshare are protected by copyright, with all rights reserved, unless otherwise indicated.

\title{
Effect of morphological state of graphene on mechanical properties of nanocomposites
}

\section{PLEASE CITE THE PUBLISHED VERSION}

http://dx.doi.org/10.1007/s10853-016-9722-0

\section{PUBLISHER}

(C) Springer Verlag (Germany)

\section{VERSION}

AM (Accepted Manuscript)

\section{PUBLISHER STATEMENT}

This work is made available according to the conditions of the Creative Commons Attribution-NonCommercialNoDerivatives 4.0 International (CC BY-NC-ND 4.0) licence. Full details of this licence are available at: https://creativecommons.org/licenses/by-nc-nd/4.0/

\section{LICENCE}

CC BY-NC-ND 4.0

\section{REPOSITORY RECORD}

Bayrak, Osman, Mariana lonita, Emrah Demirci, and Vadim V. Silberschmidt. 2019. "Effect of Morphological State of Graphene on Mechanical Properties of Nanocomposites”. figshare. https://hdl.handle.net/2134/20092. 


\section{Effect of morphological state of graphene on mechanical properties of nanocomposites}

Osman Bayraka, Mariana Ionitab, Emrah Demircia, Vadim V. Silberschmidta1

aWolfson School of Mechanical and Manufacturing Engineering, Loughborough University, Loughborough, LE11 3TU, UK

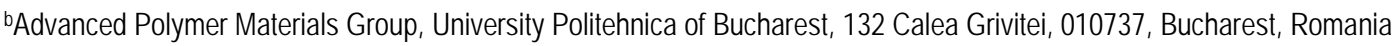

\section{Abstract}

In the last decade, graphene has emerged as one of the best-performing reinforcement materials for nanocomposites. Incorporation of graphene into polymer results in a nanocomposite with a new microstructure responsible for its enhanced features. A morphological state of graphene flakes is one of the factors that govern formation of microstructure. Studies showed that graphene-oxide (GO) flakes can be found either as fully exfoliated or intercalated in polymerbased nanocomposites. While traditional parameters are commonly taken into consideration in theoretical assessment of properties of composites by means of micromechanical models, the morphological state is often ignored. This research aims to investigate the effect of morphological state of GO flakes on stiffness of nanocomposites with widely used micromechanical models, e.g., rule of mixtures, Hui-Shia and Halpin-Tsai. Pure sodium alginate and nanocomposites on its basis reinforced with 1.0 and $2.5 \mathrm{wt} \% \mathrm{GO}$ were used in the study. Parameters required for modelling were quantified with microstructural characterisation. Micromechanical models were adapted to account for the morphological state of intercalation observed in the characterisation study. Tensile experiments were employed to assess the adopted models, and the effect matrix stiffness, GO thickness, spacing of intercalates as well as the Poisson's ratio and stiffness of inter-flake polymer layers was studied.

Keywords: Graphene; Nanocomposites; Morphology; Elastic properties; Modelling

\section{Introduction}

Excellent properties of graphene in many areas has been emphasised by specialists [1]: it outperforms many other nano-reinforcements in terms of boosting mechanical performance of nanocomposites [2,3]. The main reasons behind this are its strong carbon-carbon covalent bonds 
and an inherently very large surface-to-volume ratio. Compared to other types of nanoparticles, graphene platelets can have a much larger surface area[4], which plays a key role in load transfer.

Pristine graphene is formed of a nearly defect-free, one-atom layer lattice of pure carbon atoms, bonded to each other with $\mathrm{sp}^{2}$ hybridization. Other forms of graphene such as graphene oxide (GO), functionalized GO and graphene fluoride are also known. Before a synthesis method of pristine graphene in bulk quantities was realized [5], its other forms, especially $\mathrm{GO}$, have been intensely used for manufacturing of graphene-based nanocomposites. As a result, many advances have been made in manufacturing of GO-reinforced nanocomposites. Thanks to its compatibility with polymers, GO is still widely researched and manufactured for reinforcement of polymer nanocomposites. A type of polymer is also an important matter to consider for compatibility with oxidized graphene. Ionita et al. [6] analysed alginate (Alg)-based GO nanocomposites (GO-Alg) and found good compatibility between functional groups of GO and alginate. GO-Alg nanocomposites are the main focus of this study.

To optimise performance of nanocomposites, various fractions of graphene nanocomposites are usually employed. While numerical tools, such as, molecular dynamics [7] and finite-element simulations [8], can be preferred for design and analysis, some existing theoretical models of composites can be used for practical calculations. Some of these models preferred in graphenenanocomposite research are the rule of mixtures (RoM), Halpin-Tsai (HT) and Mori-Tanaka (MT) methods[9]. The RoM, which was originally developed for composites reinforced with continuous constituents, was modified by Padawer and Beecher [10] for discontinuous planar-particle composites. May et al. [11] investigated reinforcement efficiency of pristine graphene flakes employing a modified RoM (MRoM), While Yousefi et al. [12] used the RoM to compare the performance assessment of GO/polyurethane nanocomposites with those in literature. Voigt and Reuss approximations of the RoM were used to provide upper and lower bounds for the Young's modulus of nanoparticle-reinforced nanocomposites. Many studies employed the HT 
micromechanical model that was originally formulated for nanocomposites both with unidirectional and randomly oriented particles [13] for prediction of the Young's modulus of graphene nanocomposites [12,14-16]. In this study, only the version for unidirectional alignment is considered since the microstructural analysis demonstrated that reinforcements are preferentially aligned [6]. Another analytical model, by Tandon and Weng (TW), which was derived from the MT method [17], is also used in the literature for calculation of the Young's modulus of graphenereinforced nanocomposites $[9,18,19]$. Hui and Shia (HS) obtained simple closed-form expressions based on the TW method [20]; their method can be used to implement the MT approach in prediction of the elasticity modulus of graphene-nanocomposites [21]. The HS scheme assumes flakes as aligned in the matrix.

Young's moduli, volume fractions, orientations and aspect ratios of nano-reinforcement are the main parameters used in the analytical models to predict the effective stiffness of the nanocomposite. In these analytical models, linear elasticity of the phases is assumed and stress transfer is considered to be continuous throughout the interphases. Also, the models assume that the reinforcement flakes are homogeneously dispersed and fully exfoliated. However, depending on a manufacturing method and a type of materials constituting the nanocomposite, morphology of graphene flakes can differ. Barrett et al. [22] studied an effect of molecular structure of three different polymer matrices on mechanical performance and physical changes of their nanocomposites reinforced with reduced GO (rGO). They applied the same preparation method for all the nanocomposites, but while rGO dispersed as exfoliated in a polyhydroxyoctanoate matrix, it exhibited an intercalated morphology in polyhydroxyoctenoate. According to [23], nanocomposites produced with two different methods, namely, solvent mixing and melt blending, yield different morphologies of graphene flakes. While the solvent-mixing method gave a microstructure with homogeneously exfoliated and preferentially aligned graphene distribution in the matrix, the melt-blending method produced an intercalated and random distribution of flakes. Mechanical tests in these studies indicated from the effect of the resulting microstructures with 
different morphologies of the reinforcements: nanocomposites with intercalated graphene flakes showed poorer mechanical performance. Hence, the theoretical prediction of mechanical properties of nanocomposites can be faulty if the morphology of the reinforcement is not taken into account.

Generally, graphene dispersion in polymer nanocomposites is classified into three main states: stacked, intercalated and exfoliated [18]. Graphene or GO flakes with a stacked morphology are characterized by presence of more than several sheets. A flake with more than 10 atomic layers is not accepted as graphene $[24,25]$. Therefore, the main morphological states of graphene (and GO) are intercalated and exfoliated. As experiments showed [22,23], morphological differences caused a major effect on mechanical performance of composites. Lower stiffness reported for composites with intercalated graphene is mainly due to the fact that reinforcement in the matrix phase has a form of intercalated clusters - sandwiches of graphene and polymer. Obviously, such clusters are weaker than graphene and their aspect ratios are smaller.

In this study, a micromechanical analysis of GO nanocomposites with intercalated morphology of reinforcement is performed. Transmission electron microscopy (TEM) images were used to assess microstructure and morphology of the studied materials. Thickness of GO flakes was found with atomic force microscopy, while effective mechanical properties were obtained with quasi-static tensile tests. For the sake of better prediction of the Young's modulus of such materials, the micromechanical models were adapted to account the intercalated morphology. The effect of matrix stiffness, GO thickness, layer spacing in intercalates as well as the Poisson's ratio and stiffness of inter-flake polymers were researched with the adapted models and a developed finite-element scheme.

\section{Experimental and modelling details}

\section{Characterization}

A previous X-ray diffractometry (XRD) study, reported in [6], showed that a characteristic peak of a GO spectrum shifted towards lower angle values for GO-Alg nanocomposites. This observation revealed that morphology of GO flakes was intercalated. For a further understanding of 
morphology and microstructure of this composite, a TEM study was conducted. To implement it, small pieces of composites were first embedded in epoxy. To obtain $\sim 100 \mathrm{~nm}$-thin sections of the samples, the materials were sliced using an ultra-microtome machine with a diamond knife. The sections were placed on copper grids and monitored using a JEOL JEM-2000FX TEM system with $100 \mathrm{kV}$ acceleration voltage of electron beams. Micrographs with magnifications at $8000 \mathrm{x}$ to 300000x were obtained. In the AFM study, a scanning probe microscope NTEGRA Aura - NTMDT (NT-MDT Co., Russia) was used to evaluate the thickness of GO. For this study, GO was dispersed in water and deposited on fresh cleaved mica substrate.

Quasi-static tensile tests were conducted on the samples that were prepared according to "Standard Practice for Cutting Film and Sheeting Test Specimen, ASTM D6287-09"; their dimensions were $50 \mathrm{~mm}$ (length) $\times 3 \mathrm{~mm}$ (width). Instron 3345 uniaxial testing system with its Bluehill ${ }^{\circledR}$ software was used to perform the tensile tests. After maintaining the gauge length of 30 $\mathrm{mm}$, parameters of the test were set according to ASTM D3039. The tests were performed with three samples for each type of material.

\section{Modelling}

The traditional micromechanical models used to calculate the Young's modulus of graphenenanocomposites consider reinforcements as fully exfoliated and homogeneously distributed. For composites with intercalated clusters of graphene, the use of such models can result in errors, as can be found in literature. For this reason, appropriate adjustments of models employed should be implemented before micromechanical analysis of such nanocomposites. Luo and Daniel [26] treated the intercalated structure of polymer-clay nanocomposites as a parallel system of platelets to calculate its Young's modulus with the RoM and use it as input parameter in the MT model. GO-polymer intercalations were treated in similar way in that study, i.e. assuming them as a system of continuous and parallel platelets. The Young's modulus of the intercalated structures $E_{\mathrm{f}}^{\mathrm{ic}}$ was calculated using the Voigt's RoM as they are composed of parallel layers of GO and polymer. Then, $E_{\mathrm{f}}^{\mathrm{ic}}$ was implemented as the Young's modulus of inclusions in such widely used 
micromechanical models as MRoM, HT and HS. The respective equations for effective moduli in these models can be introduced as follows:

$$
\begin{aligned}
E_{\mathrm{c}}^{\text {Voigt }} & =E_{\mathrm{m}} v_{\mathrm{m}}+E_{\mathrm{f}} v_{\mathrm{f}} ; \\
E_{\mathrm{c}}^{\text {Reuss }} & =\frac{E_{\mathrm{m}} E_{\mathrm{f}}}{E_{\mathrm{m}} v_{\mathrm{f}}+E_{\mathrm{f}} v_{\mathrm{m}}} ; \\
E_{\mathrm{c}}^{\text {modified }} & =E_{\mathrm{m}} v_{\mathrm{m}}+\eta_{\mathrm{mrf}} E_{\mathrm{f}} v_{\mathrm{f}},
\end{aligned}
$$

where $\eta_{\mathrm{mrf}}$ is the modulus-reduction factor:

with

$$
\eta_{\mathrm{mrf}}=1-\frac{\tanh q}{q}
$$

where

$$
\begin{gathered}
q=\frac{l}{t} \sqrt{\frac{G_{\mathrm{m}} v_{\mathrm{f}}}{E_{\mathrm{f}} v_{\mathrm{m}}}} ; \\
E_{\mathrm{c}}^{\text {Halpin-Tsai }}=E_{\mathrm{m}} \frac{1+\eta_{\mathrm{L}} v_{\mathrm{f}} \xi}{1-\eta_{\mathrm{L}} v_{\mathrm{f}}} ;
\end{gathered}
$$

$$
\begin{gathered}
\eta_{\mathrm{L}}=\frac{E_{\mathrm{f}}-E_{\mathrm{m}}}{E_{\mathrm{f}}+E_{\mathrm{m}} \xi} ; \quad \xi=\frac{2 l}{3 t} ; \\
E_{\mathrm{c}}^{\text {Hui-Shia }}=\frac{E_{\mathrm{m}}}{1-\frac{v_{\mathrm{f}}}{4}\left(\frac{1}{\xi}+\frac{3}{\xi+\Lambda}\right)},
\end{gathered}
$$

where

$$
\xi=v_{\mathrm{f}}+\frac{E_{\mathrm{m}}}{E_{\mathrm{f}}-E_{\mathrm{m}}}+3\left(1-v_{\mathrm{f}}\right) \frac{\left(1-\frac{\pi t}{2 l}\right)\left(\frac{t}{l}\right)^{2}-\frac{t}{2 l}}{\left(\frac{t}{l}\right)^{2}-1}
$$

with

$$
\Lambda=\left(1-v_{\mathrm{f}}\right) \frac{3\left(\left(\frac{t}{l}\right)^{2}+0.25\right) \frac{\pi t}{2 l}-2\left(\frac{t}{l}\right)^{2}}{\left(\frac{t}{l}\right)^{2}-1} .
$$

In these models, $E_{\mathrm{f}}$ is replaced with $E_{\mathrm{f}}^{\mathrm{ic}}$ - the Young's modulus of intercalated clusters that is defined by the Voigt approximation of the RoM:

$$
E_{\mathrm{f}}^{\mathrm{ic}}=E_{\mathrm{m}} v_{\mathrm{m}}^{\mathrm{ic}}+E_{\mathrm{f}} v_{\mathrm{f}}^{\mathrm{ic}},
$$

where $v_{\mathrm{m}}^{\mathrm{ic}}$ and $v_{\mathrm{f}}^{\mathrm{ic}}$ are the volume fractions of the matrix and $\mathrm{GO}$ in the intercalated clusters. Also, $v_{\mathrm{f}}$ and $v_{\mathrm{m}}$ are replaced with $v_{\mathrm{ic}}$ and $1-v_{\mathrm{ic}}$, respectively; $v_{\mathrm{ic}}$ is defined by 


$$
v_{\text {ic }}=v_{\mathrm{f}} \frac{d_{\mathrm{s}}^{\mathrm{ic}}}{t}
$$

where $d_{\mathrm{s}}^{\text {ic }}$ is the spacing between the graphene layers in the clusters found with either XRD or TEM. In all the equations, indices $\mathrm{f}$ and $\mathrm{m}$ stand for the matrix and flakes, respectively, while $G_{\mathrm{m}}$, $l$ and $t$ are the shear modulus of matrix, thickness and length of reinforcements, respectively. A volume fraction of the intercalated structures was calculated with Eq. (7), with geometric properties of the intercalated structures obtained from TEM images. In manufacturing of nanocomposites, the weight fraction is more broadly used than the volume one; hence, the weight fraction of GO flakes in the composites were converted into the volume fraction using

$$
v_{\mathrm{f}}=\frac{w_{\mathrm{f}} \rho_{\mathrm{m}}}{w_{\mathrm{m}} \rho_{\mathrm{f}}+w_{\mathrm{f}} \rho_{\mathrm{m}}},
$$

where $\rho$ and $w$ are the density and weight fraction, respectively.

Based on XRD studies [6] that showed that addition of GO had a minor effect on a crystalline structure of Alg, it was considered that the polymer intercalating GO had the same mechanical properties as the matrix.

To study the effect of the Poisson's ratio, a finite-element (FE) model was developed employing a MSC Marc ${ }^{\circledR}$ FE software package.. GO layers and the intercalating polymer between them were modelled with different material formulations; Solid-shell elements (type 185) were used in both cases. Thickness and length of the simulated intercalated structure were $25 \mathrm{~nm}$ and $2 \mu \mathrm{m}$, respectively, while the width was equal to one-tenth of the length. These geometric features were also used in the above theoretical models... Permanent contact between the GOs and the Alg layers was applied in FEM to represent existing covalent bonding. Before simulations, a meshconvergence study was performed; it showed that stress-strain behaviour of the model did not change considerably with the number of elements. Eventually,, simulations were implemented with the total number of elements of 5160 . Boundary conditions were applied in a way to reflect those of the uniaxial tensile test; the FE model is shown in Fig. 1. Static, large-strain analysis was performed in simulations. 


\section{Results and discussion \\ Experimental results}

The obtained AFM results (Fig. 2) indicated the presence of multiple and individual layers of GO in the sheet-like shapes with a rather non-uniform size distribution. The average thickness of individual GO sheets measured from the height profile of the AFM image was about $0.916 \mathrm{~nm}$. TEM micrographs of the nanocomposites (Figs. 3 and 4) demonstrate intercalated structures (dark areas in Figs. 3(a) and (c)), which are homogeneously distributed throughout the matrix (a light grey colour). This distribution character was observed also in other micrographs taken from different areas of the nanocomposites. High-magnification micrographs (Figs. 3(b) and (d)) were taken to find thickness of the intercalated structures; the latter cannot be defined until a magnification, at which features of single flakes become observable, is reached. This is affected by the method of TEM that depicts a slice of specimen through its thickness, not an image of its surface: it gives a projected view. In other words, if a flake is not normal but rather inclined to the plane of the specimen's slice used for TEM, a contour of the flake would be observed rather than its thickness. Hence, thicknesses measurements made at insufficient magnifications would be misleading. The solution to this is attaining a higher magnification, at which the edges of single graphene sheets can be realized; only in this case the true thickness of intercalations can be obtained for flake-reinforced nanocomposites. In the micrographs that were obtained at 200000x magnifications (Figs. 3 (b) and (d)), thin edges of GO sheets are seen. Therefore, the thickness of intercalations from images with this magnification is considered and used as the relevant parameter in the theoretical calculations. The magnitude of thickness obtained in this way was some $25 \mathrm{~nm}$.

Even higher magnification (Fig. 4(e)) was used to assess intercalation spacing. As suggested by the XRD results [6], GO intercalation spacing was found to be $\sim 1.7 \mathrm{~nm}, 1.742 \mathrm{~nm}$ and $1.719 \mathrm{~nm}$ for $1.0 \mathrm{wt} \%$ and $2.5 \mathrm{wt} \%$ GO-Alg, respectively.

In TEM (Fig. 3) and AFM images (Fig. 2), it is seen that GO diameter varies from $100 \mathrm{~nm}$ to several micrometres. However, since large-size flakes are highly dominant, the magnitudes of 
diameter (or length) of the small-size flakes were not considered. Therefore, a mean flake diameter was determined as $2.0 \mu \mathrm{m}$ from these images.

Results of the performed tensile tests are shown together in a stress-strain diagram (see Fig. 5); values of the Young's modulus, yield stress and yield strain calculated from the curves in Fig. 5 are given in Table 1. Apparently, even a lower content of GO resulted in a considerable increase in toughness of the nanocomposite while the higher amount of GO made it brittle.

\section{Modelling results}

\section{Examination of adapted theoretical models}

The Young's moduli of the nanocomposites were estimated employing the micromechanical models, which were adapted as explained in Section 2.2. To assess the effect of intercalation, the estimations were also made with traditional micromechanical models. These models assume that the flakes are homogeneously dispersed and the stress transfer happens through the interface. Since the functional groups of both the matrix and GO lead to covalent bonding on the interfaces, it can be assumed that the stress transfer occurs through the interfaces. Also, TEM micrographs at 15000x magnifications (Figs. 3(a) and (c)) showed a homogeneous distribution of flakes. The Young's modulus of Alg matrix was found as $4.75 \mathrm{GPa}$ from the tensile tests (see Table 1;. the stiffness level for a single GO sheet is given in literature as $145.3 \mathrm{~N} \mathrm{~m}^{-1}$ [27]. The thickness of GO that was used in this study was found from AFM to be $0.916 \mathrm{~nm}$ (see Section 3.1). Using this thickness, the Young's modulus of GO was found as $158.7 \mathrm{GPa}$. The average flake diameter was assessed as $\sim 2.0 \mu \mathrm{m}$ from the AFM and TEM images. The density of Alg film was calculated from [28] as $1.0 \mathrm{~g} \mathrm{~cm}^{-3}$, that of $\mathrm{GO}$ is given as $1.8 \mathrm{~g} \mathrm{~cm}^{-3}$ by [27]. Using Eq. (8), the volume fractions $v_{\mathrm{f}}$ of GO were calculated as $0.56 \%$ and $1.4 \%$ for $1.0 \mathrm{wt} \%$ and $2.5 \mathrm{wt} \%$ GO-Alg, respectively.

Predictions of the Young's modulus, using the traditional formulations of micromechanical models as well as those adjusted to account for platelets' morphology are shown in Figs. 6(a) and (b), respectively. Apparently, the latter models approximate the test results better than the traditional 
ones do. It was also found that the MRoM is more suitable than the HS and HT models for predicting the modulus of GO-Alg nanocomposites.

Importance of considering the intercalation phenomenon for prediction of the Young's modulus of nanocomposites was studied additionally by taking several parameters into consideration. The parameters of interest are effects of matrix stiffness, stiffness of inter-flake polymers, the Poisson's ratio of inter-flake polymers, layer-spacing of intercalates and GO thickness. These parameters were investigated using the morphology-adjusted MRoM (maMRoM), as it worked better for our samples than the modified HS and HT models. The deviations of composite moduli mentioned in the sections below were related to the respective maMRoM values.

\section{Effect of matrix stiffness}

The matrix modulus is one of the parameters defining the effect of reinforcement in composites. As the Young's modulus of matrix material increases, while all other parameters are constant, the effect of reinforcement decreases. A study of this effect was aimed to reveal importance of considering the intercalation phenomena in predicting the effective Young's modulus of nanocomposites. A chart in Fig. 7 shows deviation of the estimated Young's modulus of nanocomposites as a function of modulus of the matrix material for different volume fractions of GO, ranging from $0.1 \%$ to $10 \%$. To create the chart, thickness and length of the intercalated structures, thickness of GO, and spacing between layers were kept constant with the values of those parameters given in the previous section. As can be seen from the graph, the deviation diminishes with the increasing matrix modulus. For matrixes with the modulus of $5 \mathrm{GPa}$, the deviation is less than $20 \%$, For stiffer matrices with modulus of $10 \mathrm{GPa}$ and higher, it becomes less important to consider the intercalation effect since the deviation drops below $10 \%$. Apart of the lowest volume fraction - $0.1 \%$, the latter does not seem to have a major role in this deviation. Therefore, it can be concluded that for GO-nanocomposites with matrix stiffness of $10 \mathrm{GPa}$ and above, and with any volume fraction of $\mathrm{GO}$, implementation of the account for intercalation is not necessary. However, for the nanocomposites with a softer matrix it is important for an adequate 
assessment of effective elastic properties.

\section{Effect of inter-flake matrix stiffness}

Thin layers of matrix intercalated into the GO sheets can have their modulus different from that of the base matrix due to potential changes in their microstructure related to their small thickness. Therefore, the next stage of our study is focused on quantification of this effect. The respective results are presented in Fig. 8 for different volume fractions of $G O$ and varying stiffness of the matrix (all the other parameters were fixed). The obtained results vividly demonstrate that for any analysed volume fraction of GO platelets, the variation of the inter-flake matrix modulus from $50 \%$ to $+50 \%$ has a marginal effect on the estimated effective stiffness. Thanks to the linear character of the curves, it can be predicted that even a $100 \%$ variation of the inter-flake matrix stiffness from that of the base matrix would not cause a considerable change in the overall composite stiffness. Hence, a modified micromechanical approach could safely use the assumption that layers between graphene sheets in intercalated platelets have the same stiffness as the base polymer matrix.

\section{Effect of Poisson's ratio of inter-flake matrix}

As the modulus of inter-flake matrix may differ from that of the base matrix, its Poisson's ratio can change too. To characterise the sensitivity of the composite's modulus to the changes in the interflake's Poisson's ratio, an FEM of the intercalated structure developed as explained in Section $\mathbf{2 . 2}$ was used to perform tensile-test simulations to assess the Young's moduli of the intercalated structures (flakes) with different moduli and Poisson's ratios of the inter-flake matrix. While the length, thickness and spacing between layers of intercalated structures were kept constant as 2.0 $\mu \mathrm{m}, 25 \mathrm{~nm}$ and $1.7 \mathrm{~nm}$, respectively, the Poisson's ratio of the inter-flake matrix was varied from 0.1 to 0.49 ; calculations were carried out for matrix moduli of $0.1,4.75$ and $10 \mathrm{GPa}$. The GO thickness was kept the same at $0.916 \mathrm{~nm}$, while the mechanical properties of the GO layers were fixed at the values given in previous sections. A change in Young's moduli of the intercalated structures for three different levels of the matrix modulus is plotted in Fig. 9 depending on the 
Poisson's ratio. The maximum deviation is found for the high-modulus matrix, and it is less than $3 \%$. Such a small deviation in properties of flakes would have even a lower effect on the effective modulus of nanocomposites composite. Therefore, it can be concluded that the changes in Poisson's ratio of the inter-flake matrix does not have a considerable effect on the nanocomposites' moduli of elasticity. It can be omitted when doing the stiffness prediction of GOpolymer nanocomposites.

\section{Effect of layer spacing}

As explained in the previous sections, layer spacing in flakes (GO platelets) can be measured with either XRD or TEM. Apparently, measuring at such small scales requires high precision. As a result, some small measurement errors can affect moduli estimation. In order to quantify sensitivity of such estimations to the potential errors in measurements of layer spacing, values of the Young's modulus were calculated for different volume fractions of $\mathrm{GO}$ and matrix moduli. Other parameters were held constant at the levels described above. Deviations of the nanocomposites' modulus in this study are plotted against the change in layer spacing in Fig. 10. The measurement error was considered to be between $-20 \%$ and $+20 \%$ of the original value. The results show that for volume fractions of GO up to $1.0 \%$ of $\mathrm{GO}$ and any matrix modulus the composite modulus is not affected considerably. However, as the volume fraction increases and the matrix moduli decreases, the composite modulus gets more sensitive to measurement errors for the layer spacing and its deviations can exceed $50 \%$.

\section{Effect of thickness of GO layers}

The thickness of GO layers (sheets), measured with either AFM or XRD, is reported to vary between 0.8 and $1.2 \mathrm{~nm}$ [29]. Knowing the level of stiffness of a single GO sheet $-145.3 \mathrm{~N} \mathrm{~m}^{-1}$, one can calculate its Young's modulus depending on the thickness. Considering that GO thickness does not vary significantly for the same manufacturing method, a value found in the literature can be used in the calculations. However, this assumption may lead to misestimating the Young's moduli of the studied nanocomposites. Keeping the length, thickness and layer 
spacing of intercalated structures constant (as given in the previous section) and considering the reference GO thickness as $1 \mathrm{~nm}$ (the mean value of data reported in [29]), assessment of the Young's modulus was carried out for varying levels of the volume fractions of $G O$ and matrix modulus. The obtained results are presented in Fig. 11. The range of deviations in the GO thickness was assumed as $\pm 20 \%$ to match the reported interval of $0.8-1.2 \mathrm{~nm}$. Since the layer spacing was kept constant, the character of the effect is opposite in this case. The results show that, similar to the case of layer spacing, as the Young's modulus of matrix decreases and volume fraction of GO increases, precise measurement of GO thickness gains importance for adequate assessment of the effective properties of the studied nanocomposites.

\section{Conclusion}

The effect of intercalated morphology of GO on nanocomposites with Alg matrix was analysed based on experimental, microstructural and numerical studies. Pure Alg polymer and nanocomposites with 1.0 and $2.5 \mathrm{wt} \% \mathrm{GO}$ reinforcement were characterized with TEM and tested with tensile machine.

Microstructural analysis on the nanocomposites with XRD and TEM suggested that GO flakes were intercalated rather than exfoliated. Traditional micromechanical models - HS, HT and MRoM - were adjusted to account for the intercalated structure. The Young's modulus of the composites, calculated with both the original versions and ones accounting for the GO morphology, were compared with the experimental findings. The results showed that the adjusted models predicted the Young's modulus much better than the traditional models. Among these micromechanical models incorporating the effect of GO morphology, the MRoM provided more accurate results than $\mathrm{HT}$ and $\mathrm{HS}$.

Using this version of the MRoM, the factors making the consideration of intercalation phenomena important were revealed and the effects of some parameters that can affect the effective properties, including variations in some parameters as well as potential measurement errors, were assessed. It was shown that nanocomposites with intercalated GO flakes with matrix modus of less than $10 \mathrm{GPa}$ should be assessed with the adjusted models in order to have accurate 
predictions. The effect of mechanical properties of the inter-flake matrix were quantified, and it was found that potential changes in the Poisson's ratio and the modulus of the inter-flake matrix did not have a considerable effect on the overall modulus of the studied nanocomposites. On the other hand, measurement errors in layer spacing and GO thickness could become important with the increase in the fraction of the flakes and the decrease of the matrix modulus. The diagrams of changes in the effective elastic modulus obtained in this study for various parameters can help to decide the suitability of the micromechanical schemes - traditional or adjusted -for its prediction for GO-reinforced nanocomposites.

\section{Acknowledgement}

The authors thank Dr Zhaoxia Zhou in Department of Materials at Loughborough University for her contribution to obtaining TEM micrographs. 


\section{REFERENCE}

[1] Allen MJ, Tung VC, Kaner RB (2010) Honeycomb Carbon: A Review of Graphene, Chem Rev 110:132-145.

[2] Rafiee MA, Rafiee J, Wang Z, Song HH, Yu ZZ, Koratkar N (2009) Enhanced Mechanical Properties of Nanocomposites at Low Graphene Content, Acs Nano 3:3884-3890.

[3] Li B and Zhong WH (2011) Review on polymer/graphite nanoplatelet nanocomposites, J Mater Sci 46:5595-5614.

[4] Jang BZ and Zhamu A (2008) Processing of nanographene platelets (NGPs) and NGP nanocomposites: a review, $J$ Mater Sci 43:5092-5101.

[5] Hernandez Y, Nicolosi V, Lotya M, Blighe FM, Sun ZY, De S, et al. (2008) High-yield production of graphene by liquid-phase exfoliation of graphite, Nat Nanotechnol 3:563-568.

[6] Ionita M, Pandele MA, Iovu H (2013) Sodium alginate/graphene oxide composite films with enhanced thermal and mechanical properties, Carbohyd Polym 94:339-344.

[7] Lee W, Jang S, Kim MJ, Myoung JM (2008) Interfacial interactions and dispersion relations in carbon-aluminium nanocomposite systems, Nanotechnology 19:

[8] Mortazavi B, Baniassadi M, Bardon J, Ahzi S (2013) Modeling of two-phase random composite materials by finite element, Mori-Tanaka and strong contrast methods, Compos Part B-Eng 45:1117-1125.

[9] Hu KS, Kulkarni DD, Choi I, Tsukruk VV (2014) Graphene-polymer nanocomposites for structural and functional applications, Prog Polym Sci 39:1934-1972.

[10] Padawer GE and Beecher N (1970) On Strength and Stiffness of Planar Reinforced Plastic Resins, Polym Eng Sci 10:185-192.

[11] May P, Khan U, O'Neill A, Coleman JN (2012) Approaching the theoretical limit for reinforcing polymers with graphene, J Mater Chem 22:1278-1282.

[12] Yousefi N, Gudarzi MM, Zheng QB, Lin XY, Shen X, Jia JJ, et al. (2013) Highly aligned, ultralarge-size reduced graphene oxide/polyurethane nanocomposites: Mechanical properties and moisture permeability, Compos Part a-Appl S 49:42-50.

[13] Halpin JC and Kardos JL (1976) Halpin-Tsai Equations - Review, Polym Eng Sci 16:344-352.

[14] Liang JJ, Huang Y, Zhang L, Wang Y, Ma YF, Guo TY, et al. (2009) Molecular-Level Dispersion of Graphene into Poly(vinyl alcohol) and Effective Reinforcement of their Nanocomposites, Adv Funct Mater 19:2297-2302.

[15] Kong JY, Choi MC, Kim GY, Park JJ, Selvaraj M, Han M, et al. (2012) Preparation and properties of polyimide/graphene oxide nanocomposite films with Mg ion crosslinker, Eur Polym J 48:1394-1405.

[16] Liu HH, Hou LC, Peng WW, Zhang Q, Zhang XX (2012) Fabrication and characterization of polyamide 6-functionalized graphene nanocomposite fiber, $J$ Mater Sci 47:8052-8060.

[17] Tandon GP and Weng GJ (1984) The Effect of Aspect Ratio of Inclusions on the Elastic Properties of Unidirectionally Aligned Composites, Polym Composite 5:327-333.

[18] Potts JR, Shankar O, Du L, Ruoff RS (2012) Processing-Morphology-Property Relationships and Composite Theory Analysis of Reduced Graphene Oxide/Natural Rubber Nanocomposites, Macromolecules 45:6045-6055.

[19] Hu K KD, Choi I, Tsukruk VV (2014) Graphene-polymer nanocomposites for structural and functional applications, Prog Poly Sci 39:1934-1972. 
[20] Hui CY and Shia D (1998) Simple formulae for the effective moduli of unidirectional aligned composites, Polym Eng Sci 38:774-782.

[21] Bandla S and Hanan JC (2012) Microstructure and elastic tensile behavior of polyethylene terephthalate-exfoliated graphene nanocomposites, J Mater Sci 47:876-882.

[22] Barrett JSF, Abdala AA, Srienc F (2014) Poly(hydroxyalkanoate) Elastomers and Their Graphene Nanocomposites, Macromolecules 47:3926-3941.

[23] Mahmoud WE (2011) Morphology and physical properties of poly(ethylene oxide) loaded graphene nanocomposites prepared by two different techniques, Eur Polym J 47:1534-1540.

[24] Edwards RS and Coleman KS (2013) Graphene synthesis: relationship to applications, Nanoscale 5:38-51.

[25] Partoens B and Peeters FM (2006) From graphene to graphite: Electronic structure around the K point, Phys Rev B 74:0754041-07540411.

[26] Luo JJ and Daniel IM (2003) Characterization and modeling of mechanical behavior of polymer/clay nanocomposites, Compos Sci Technol 63:1607-1616.

[27] Suk JW, Piner RD, An JH, Ruoff RS (2010) Mechanical Properties of Mono layer Graphene Oxide, Acs Nano 4:6557-6564.

[28] Ribeiro ACF, Sobral AJFN, Simoes SMN, Barros MCF, Lobo VMM, Cabral AMTDPV, et al. (2011) Transport properties of aqueous solutions of sodium alginate at $29815 \mathrm{~K}$, Food Chem 125:1213-1218.

[29] Qian XD, Song L, Tai QL, Hu Y, Yuen RKK (2013) Graphite oxide/polyurea and graphene/polyurea nanocomposites: A comparative investigation on properties reinforcements and mechanism, Compos Sci Technol 74:228-234.

\section{FIGURES}

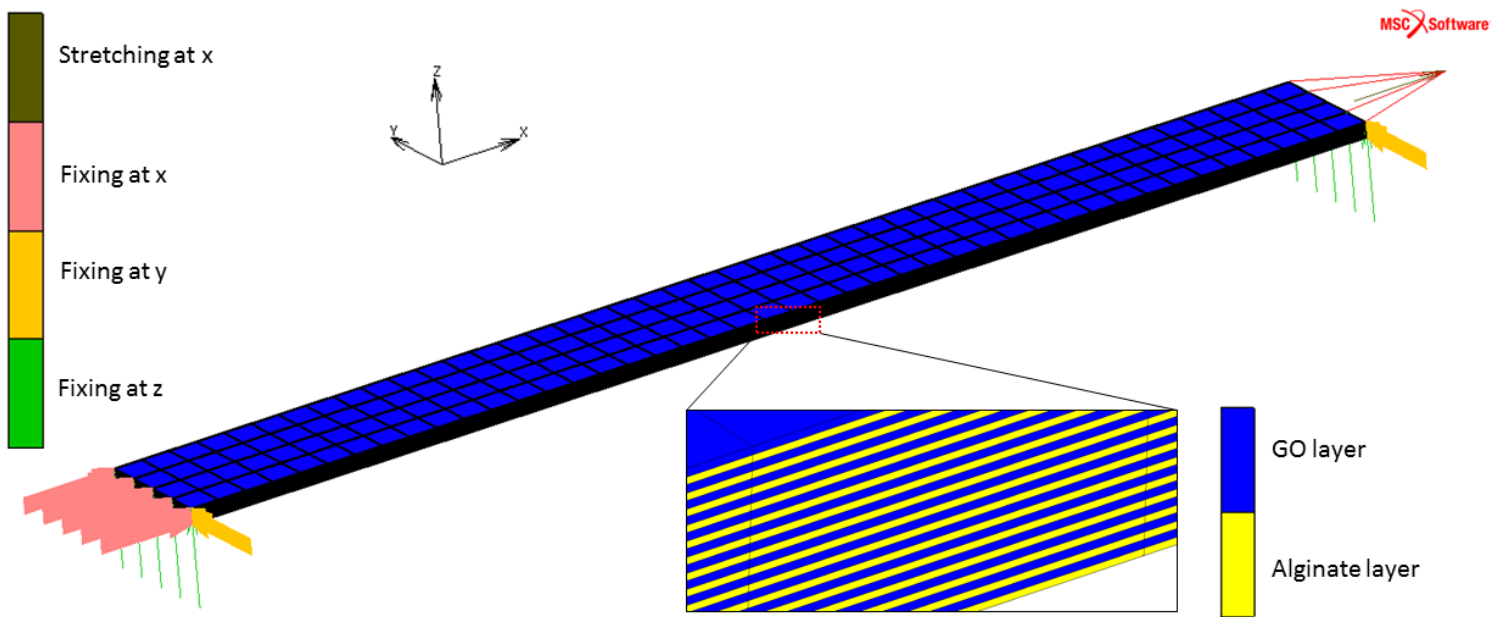

Fig. 1 Finite-element model of intercalated structure with boundary conditions and layer detail
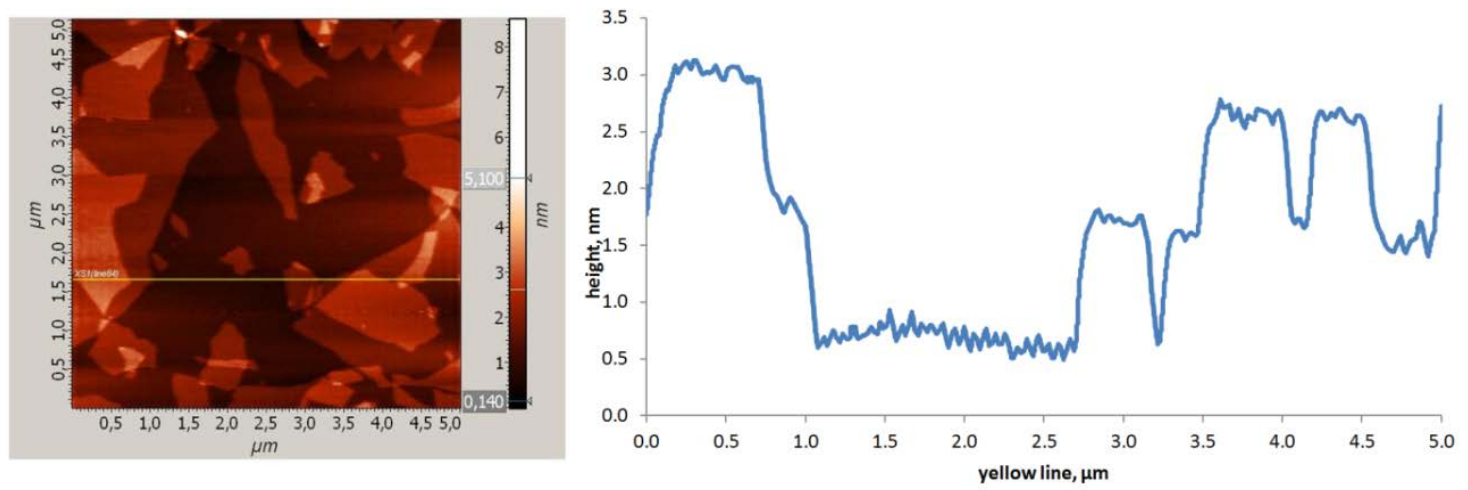
(a)

(b)

Fig. 2 AFM image (a) and height profile (b) of GO sheets along line in (a)
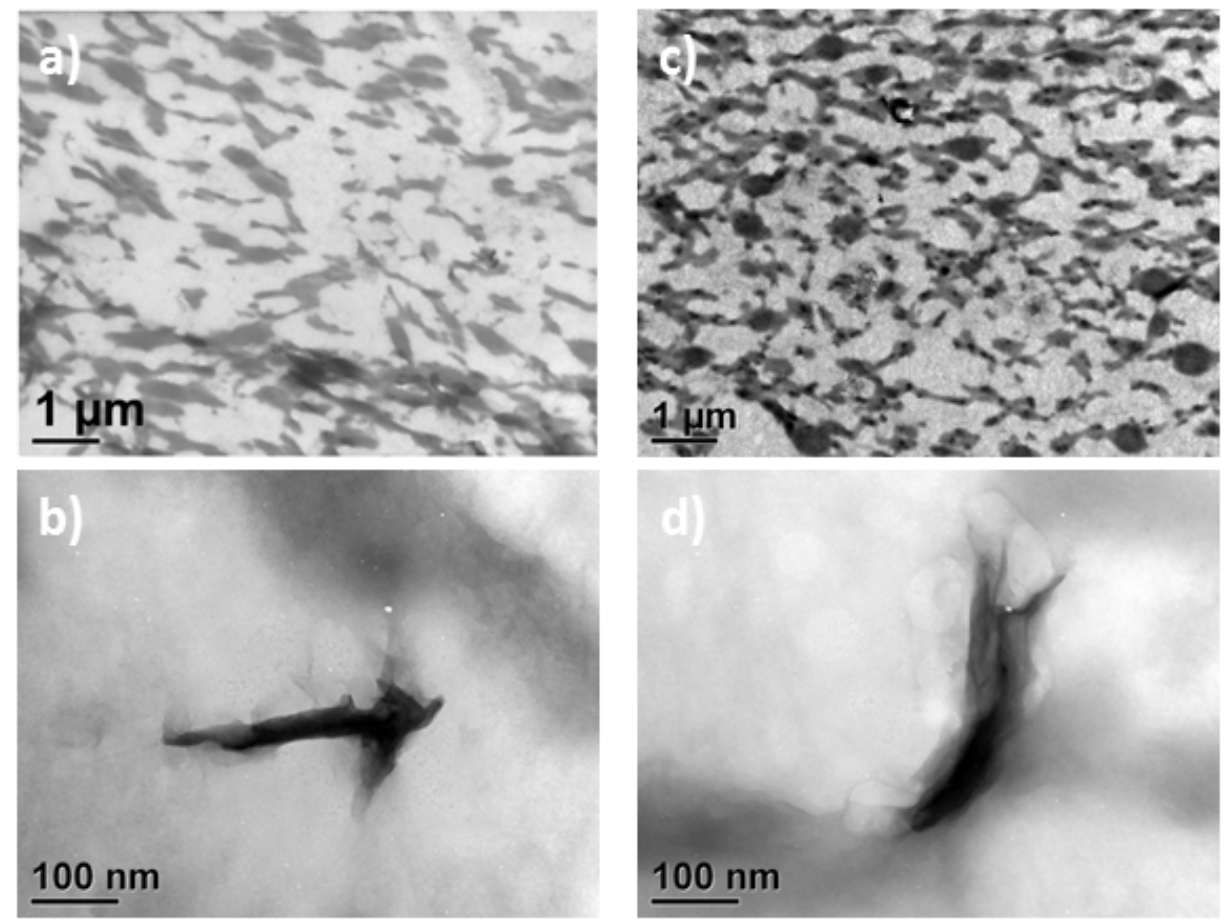

Fig. 3 TEM micrographs of GO-Alg: (a) and (b) for 1.0 wt\%; (c) and (d) for 2.5 wt\%; (a) and (c) at 15000x magnification; (b) and (d) at 200000x magnification.
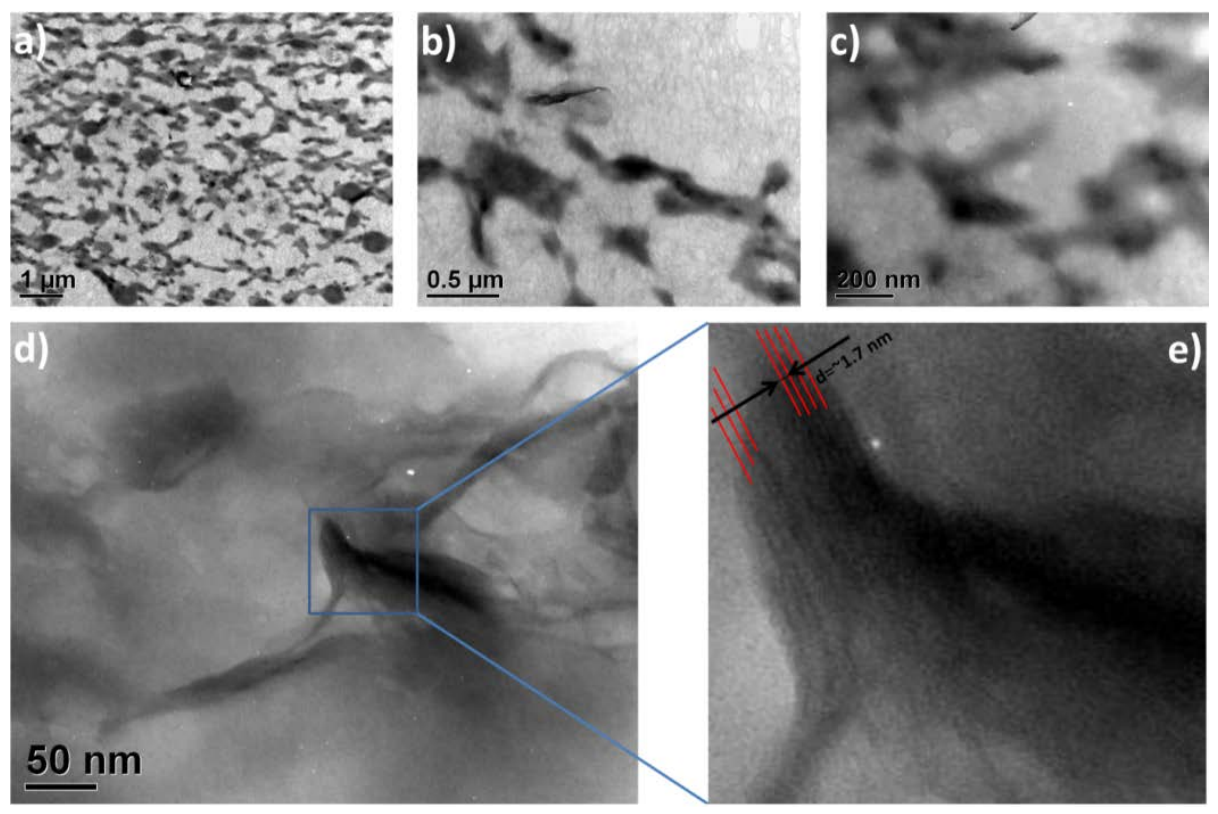

Fig. 4 TEM micrographs of $2.5 \mathrm{wt} \%$ GO-Alg nanocomposites at different magnifications:
(a) 15000 ; (b)
(b) 50000 ;
; (c) 100000;
; (d) 300000; (e) 1700000 


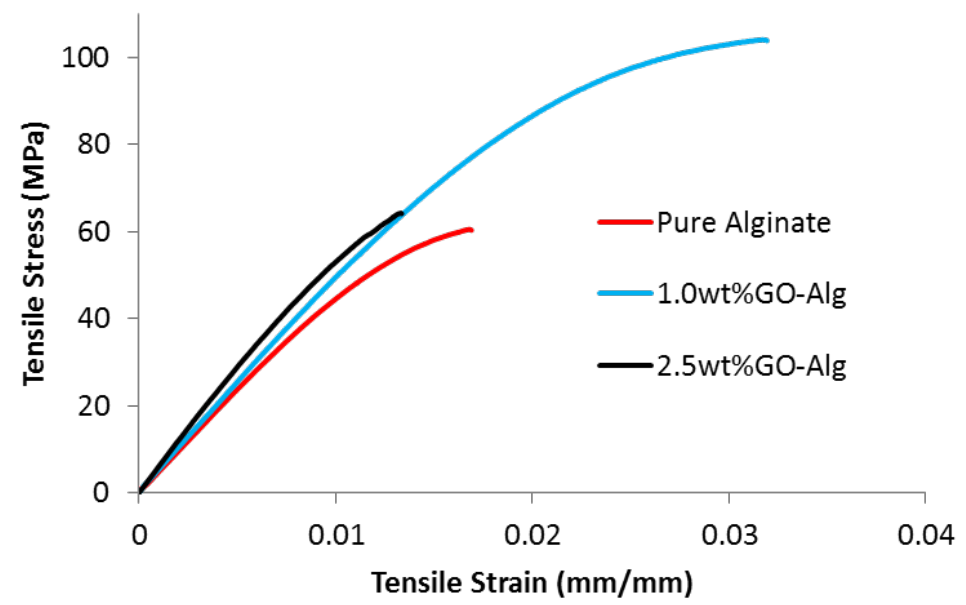

Fig. 5 Stress-strain curves of studied materials

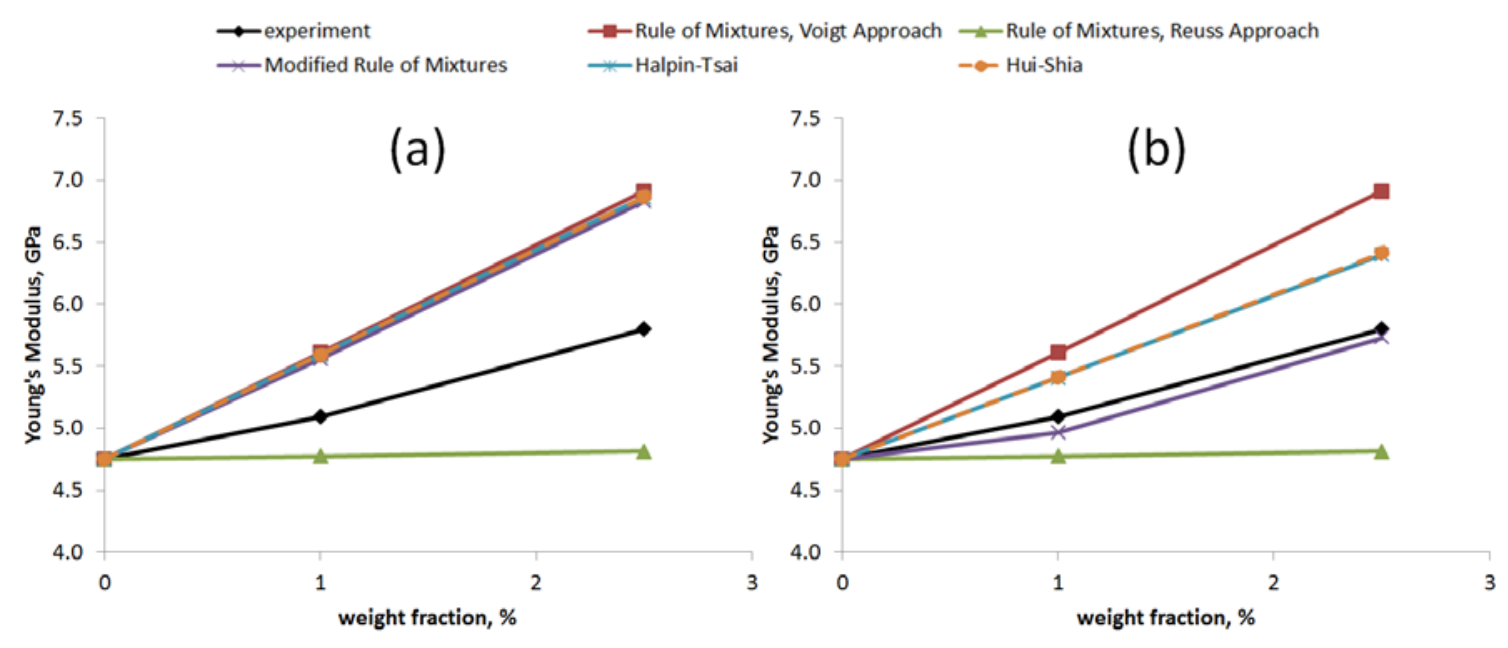

Fig. 6 Effect of weight fraction of GO on experimental and theoretical values of Young's modulus of studied materials: (a) traditional models; (b) adjusted models 


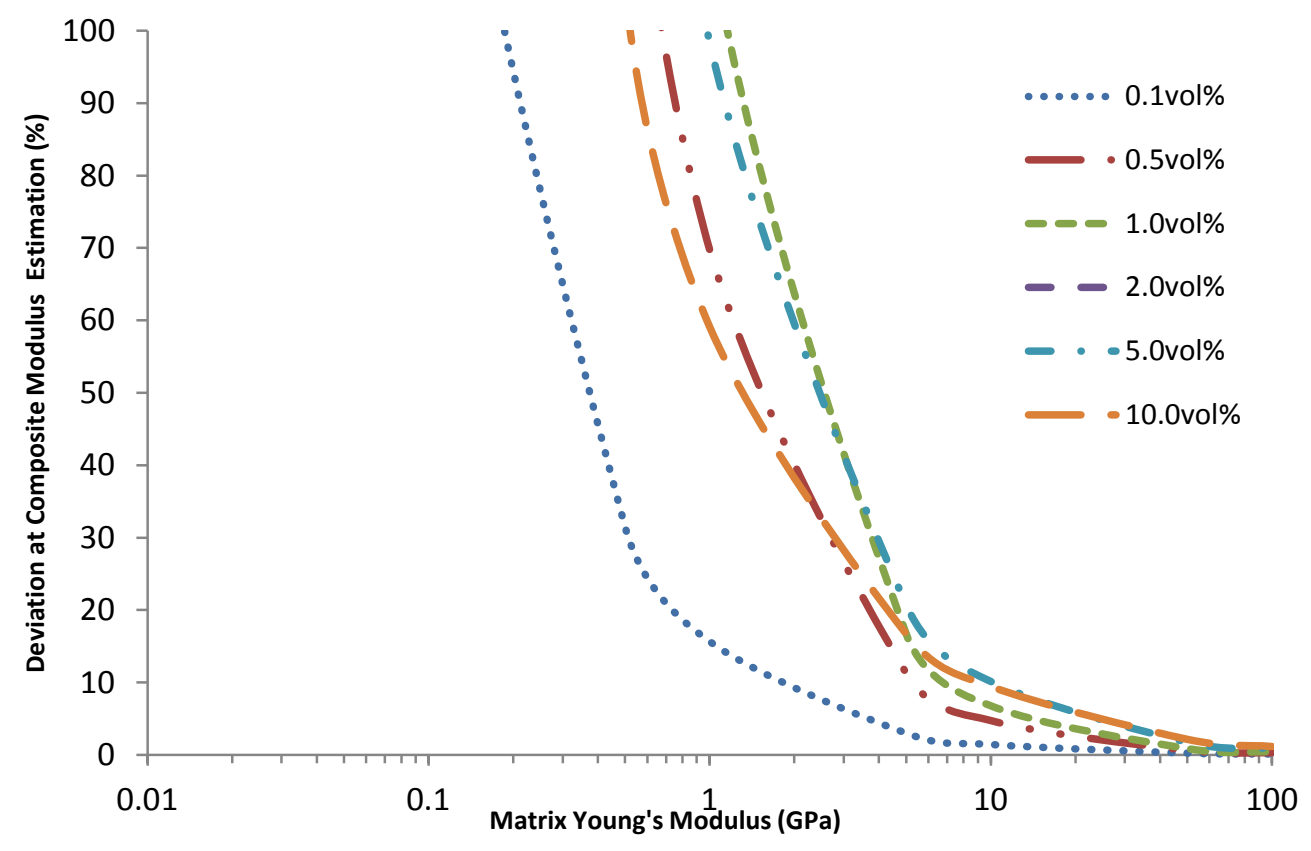

Fig. 7 Effect of Young's modulus of matrix on deviation of nanocomposite's modulus for different volume fractions of $\mathrm{GO}$.

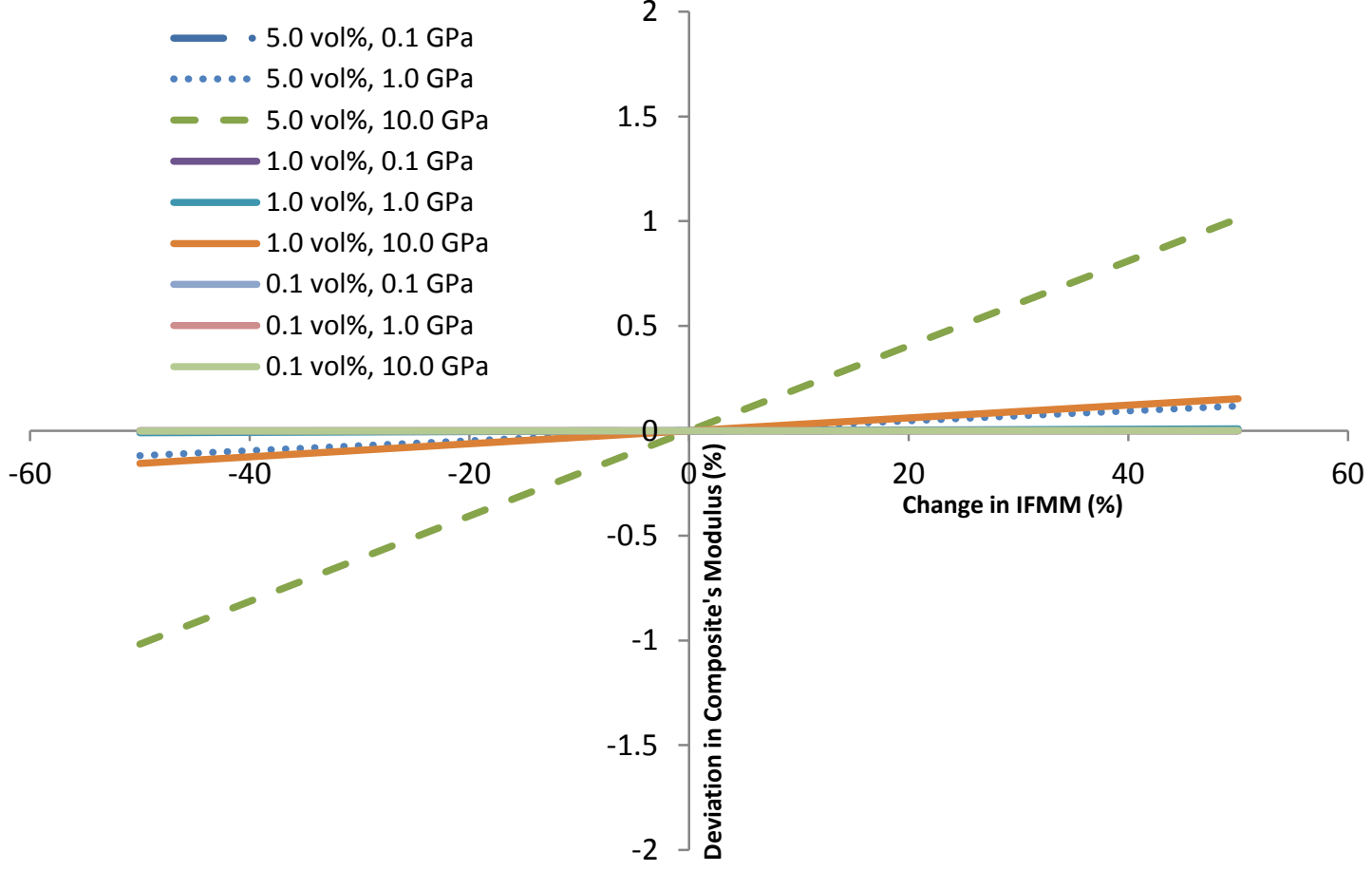

Fig. 8 Effect of inter-flake matrix modulus (IFMM) on deviation of nanocomposite's modulus for different levels of volume fraction of GO and modulus of matrixes 


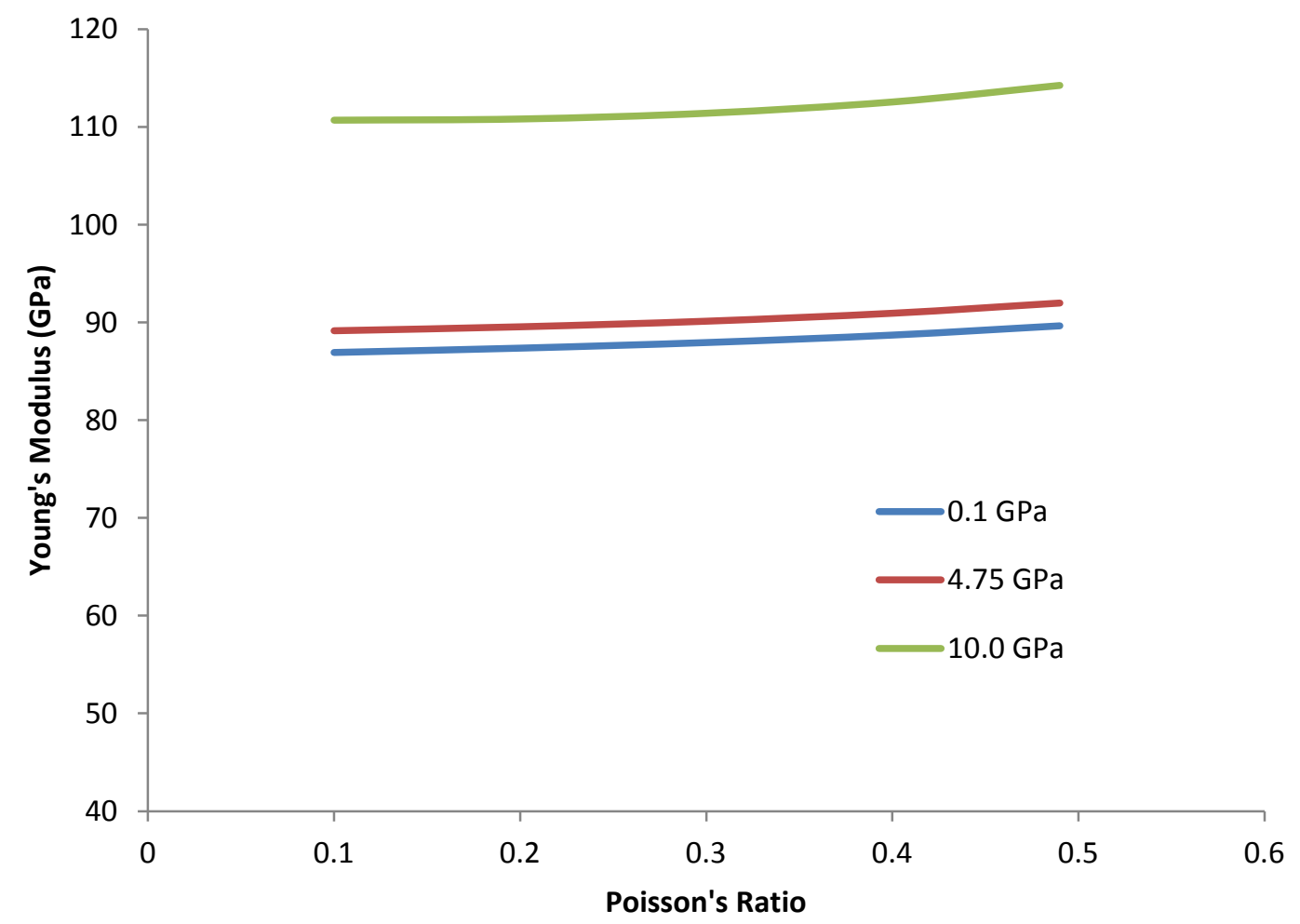

Fig. 9 Effect of Poisson's ratio on of intercalated structures on their Young's modulus for different levels of matrix modulus

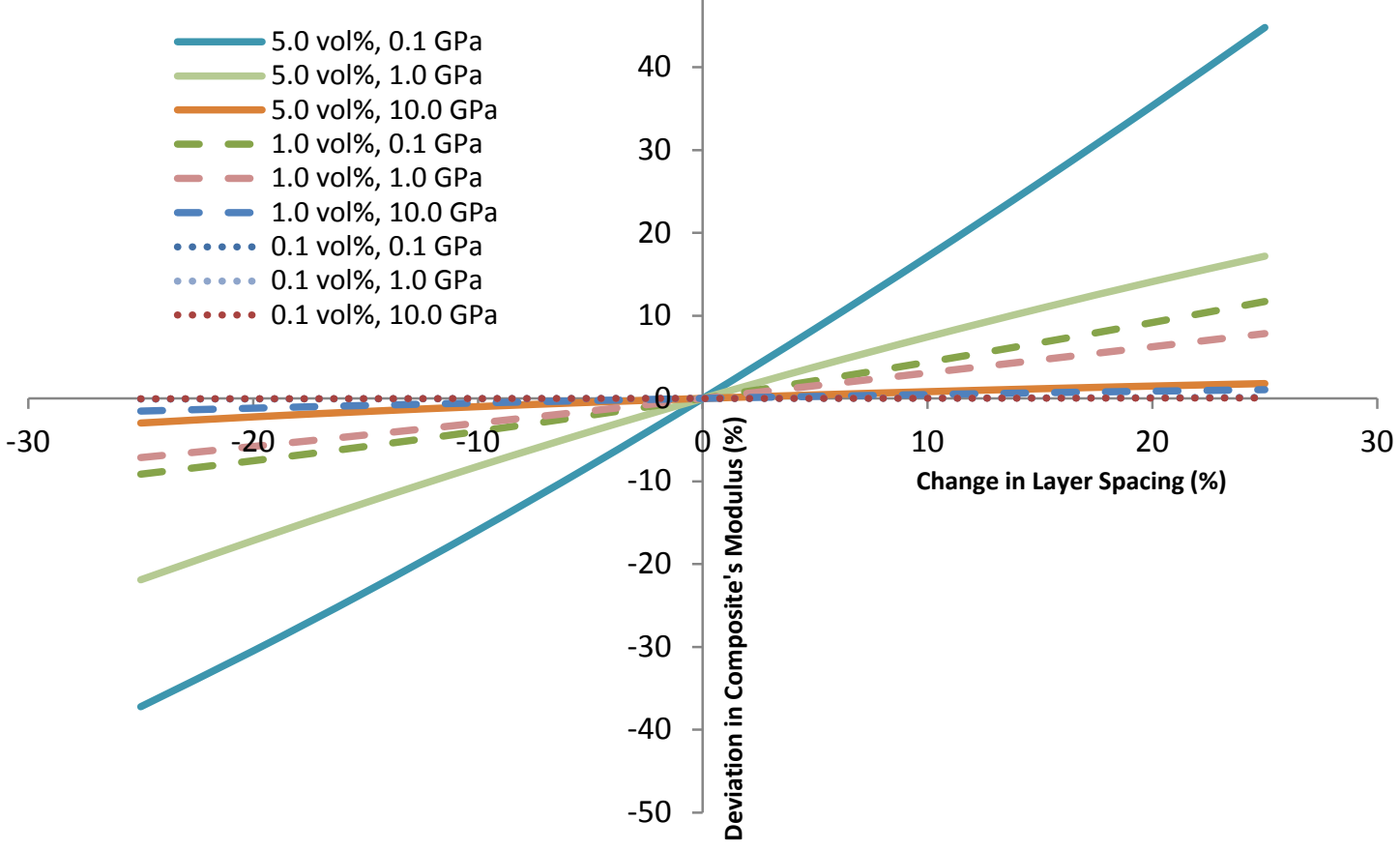

Fig. 10 Effect of layer spacing on nanocomposite's modulus for different levels of volume fraction of $\mathrm{GO}$ and matrix modulus 


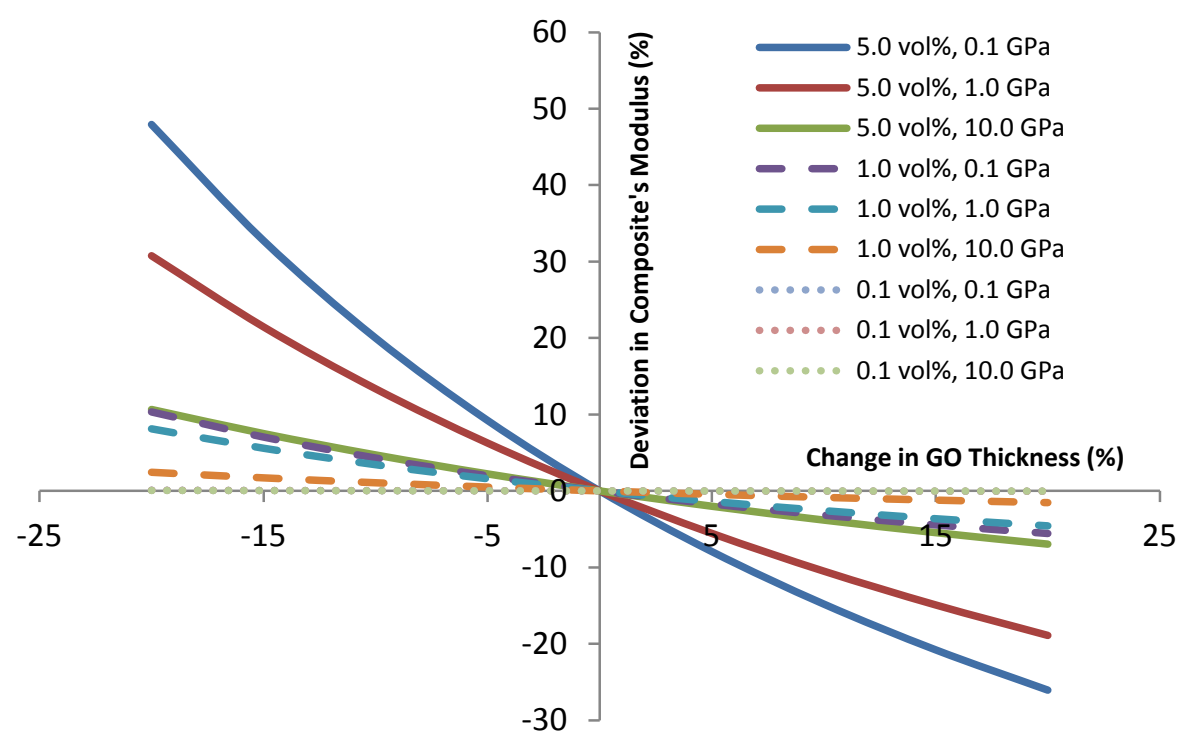

Fig. 11 Effect of GO thickness on nanocomposite's modulus for different levels of volume fraction of $\mathrm{GO}$ and matrix modulus

\section{TABLE}

Table 1 Tensile properties of materials

\begin{tabular}{|c|c|c|c|c|}
\hline Young's & $\sigma_{y}$ at offset & $\sigma_{\mathrm{y}}$ at offset & Ey at offset & $\varepsilon_{y}$ at offset \\
\hline modulus, & strain 0.001, & strain 0.002, & strain 0.001, & strain 0.002 , \\
\hline GPa & $\mathrm{MPa}$ & $\mathrm{MPa}$ & $\mathrm{mm} \mathrm{mm}^{-1}$ & $\mathrm{~mm} \mathrm{~mm}-1$ \\
\hline 4.75 & 48.8 & 55.3 & 0.011 & 0.014 \\
\hline 5.09 & 67 & 79.2 & 0.014 & 0.0175 \\
\hline 5.8 & 54.8 & 63.3 & 0.01 & 0.013 \\
\hline
\end{tabular}

